\title{
IntoTheVideos: Exploration of Dynamic 3D Space Reconstruction From Single Sports Videos
}

\author{
Xiaotong Chen \\ xchen774@ucsb.edu \\ University of California, Santa Barbara \\ Santa Barbara, California, USA
}

\author{
Misha Sra \\ sra@cs.ucsb.edu \\ University of California, Santa Barbara \\ Santa Barbara, California, USA
}

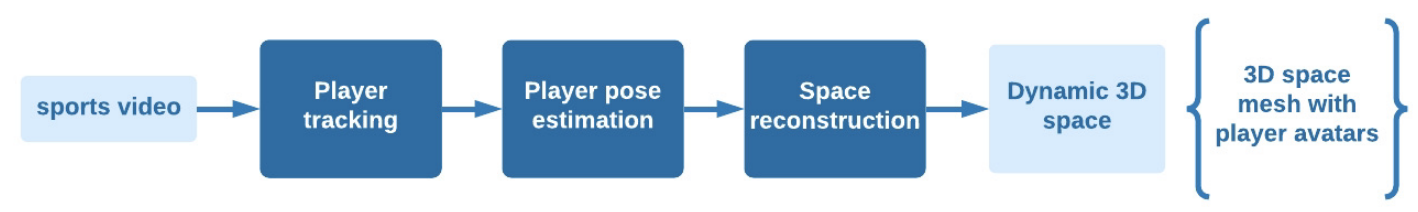

Figure 1: Pipeline overview.

\begin{abstract}
We present IntoTheVideos, a novel system that takes a sports video and reconstructs the 3D space (e.g., basketball court, football field) along with the highlighted event (e.g., making a basket, scoring a touchdown) to enable viewers to experience the sports entertainment with more freedom. Users can move around in the reconstructed 3D space and explore the event freely from different angles and distances as well as view the players in 3D. With this system, we hope to offer users a new option to enjoy sports entertainment beyond passively watching video footage potentially leading to more active fan engagement and participation.
\end{abstract}

\section{CCS CONCEPTS}

- Applied computing $\rightarrow$ Media arts; • Human-centered computing $\rightarrow$ Interaction design.

\section{KEYWORDS}

Interactive video, 3D reconstruction of dynamic scene, Sport entertainment

\section{ACM Reference Format:}

Xiaotong Chen and Misha Sra. 2021. IntoTheVideos: Exploration of Dynamic 3D Space Reconstruction From Single Sports Videos. In The Adjunct Publication of the 34th Annual ACM Symposium on User Interface Software and Technology (UIST '21 Adjunct), October 10-14, 2021, Virtual Event, USA. ACM, New York, NY, USA, 3 pages. https://doi.org/10.1145/3474349.3480215

\section{INTRODUCTION}

Modern technology has enabled various ways of sports entertainment. On one end of the spectrum, we have the more relaxed and passive options like watching broadcast sports events on television or recordings on YouTube, while on the other end of the spectrum

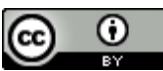

This work is licensed under a Creative Commons Attribution International 4.0 License.

UIST '21 Adjunct, October 10-14, 2021, Virtual Event, USA

(c) 2021 Copyright held by the owner/author(s).

ACM ISBN 978-1-4503-8655-5/21/10.

https://doi.org/10.1145/3474349.3480215 are the more intensive and active options like playing fantasy sports or playing sports video games in virtual reality (VR) or augmented reality (AR). However, there are limited options in the middle of the spectrum that allow users to interact with sporting events without being entirely passive or without expending too much effort. In this work, we present IntoTheVideos, a system which takes a single sports video footage and reconstructs the 3D space in the video along with the corresponding event to enable users to go into the videos and view specific events freely. Our system can handle videos with one or multiple players and is focused on footage recorded in one take (continuous shot) where the corresponding sports event takes place on a large flat surface such as in a basketball or tennis court or outside on an athletic track. The resulting 3D mesh can be imported into game engines such as Unity, allowing for easy creation of immersive first person experiences in VR or presenting the 3D game on a tabletop surface in AR. Prior work has explored the idea of interactive TV with pre-creating the 3D environments and allowing users to explore it in the third person by controlling an avatar for a lean-back experience [3]. However, unlike our system, it cannot be used with any general sports videos nor does it provide a first person immersive experience. With IntoTheVideos, we hope to diversify ways for people to enjoy sports entertainment in an immersive manner by utilizing abundantly available sports footage online.

\section{METHOD}

Reconstruction of the play space and complex events from unstructured video, like scoring a basket in basketball game, is an extremely challenging task due to the dynamics, complexity and variation of video sequences. IntoTheVideos constructs the 3D spaces from sports videos given by users in three stages (Fig. 1): 1. identify and track players in the videos, 2. estimate pose of each identified player and create player avatar, 3. reconstruct the 3D space and place player avatars. By reconstructing the space like a basketball court, and creating player avatars, we are able to offer users more freedom to enjoy the basketball video watching experience. Users can jump into the reconstructed video segments that correspond to significant events in the game such as making a basket and move around the space to view events in different angles and distances. 

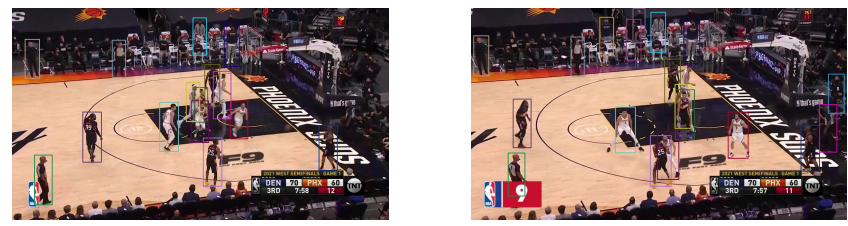

Figure 2: Tracking players in the input video $\odot$ NBA [4].

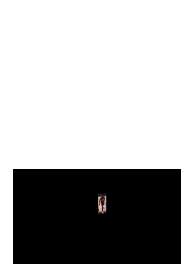

(a) Masked frame

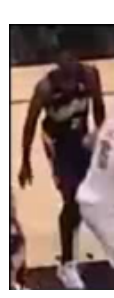

(b) Input points

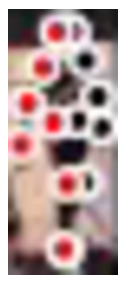

(c) Key-

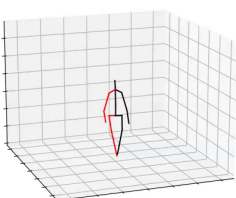

(d) Pose

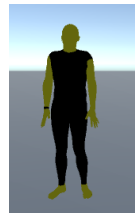

(e) Player avatar
Figure 3: Pose estimation and player avatar construction.

\subsection{Player Tracking and Pose Estimation}

Given a sports video, the first stage of our pipeline is to identify and track the players using FairMOT [8]. Fig. 4 shows two separate frames of the same basketball video, where each identified individual player is assigned a unique ID, corresponding to a unique bounding box color, that is consistent throughout the entire video. Next, we filter out individuals who could not be detected in more than $20 \%$ of the video frames to ensure that we have enough information to estimate the pose of each identified individual throughout the video in later stages of the pipeline, which should filter out most audience members and referees initially detected.

For each remaining player identified after the filtering, we construct a new video where only the pixels within the bounding boxes surrounding each individual are selected from each original input video frame (Fig. 3a). To account for frames where a particular player is not detected, such frames are substituted with their closest neighbour frames. Our system then estimates the pose of each individual in the constructed video. We first use Detectron2 [6] to localize individual keypoints (Fig. 3c), which are sent to VideoPose3D [5] to get the final 3D pose 3d. The estimated poses are used to construct a dynamic and moving $3 \mathrm{D}$ avatar. The avatar is initialized from SMPL models [2] with color matching the bounding box and the team jerseys, which helps distinguish the 3D avatars from each other while making team information available to users.

\subsection{Space Reconstruction}

After the player avatars are created, they need to be moving within the same space as seen in the original input video. Therefore, the third stage of IntoTheVideos focuses on reconstructing the static 3D space. Since most videos of interest to us are of sports played on a large flat surface (e.g., basketball court), we find 3D reconstruction methods with surface normal estimation most suitable for this task.

To perform 3D reconstruction on the rest (non-player parts) of the input video, the players detected in previous stages need to be

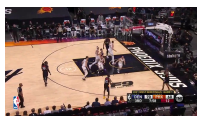

(a) Input frame

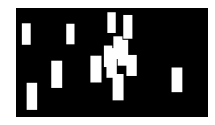

(b) Mask

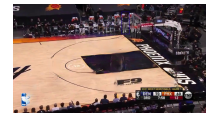

(c) Inpainted frame

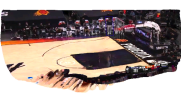

(d) Reconstruction
Figure 4: 3D space reconstruction using frames from the input video $\odot \mathrm{NBA}[4]$

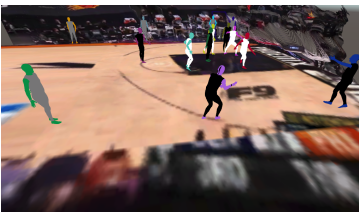

(a) Reconstructed space (w moving player avatars).
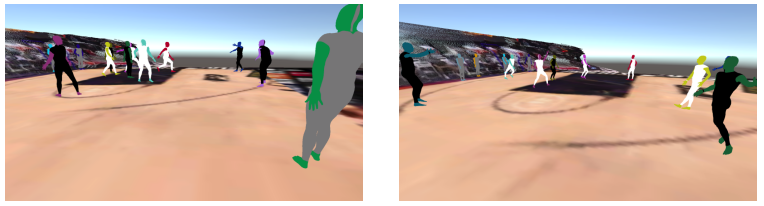

(b) Reconstructed space from a novel viewpoint. removed. We use the Partial Convolution inpainting network [1], which inpaints regions of each video frame (Fig. 4a) that are within the bounding box (Fig. 4b). Then, we use LeReS [7] to reconstruct a 3D space using the inpainted result (Fig. 4c), which correctly maintains the flatness of the ground plane (Fig. 4d).

Lastly, our system unifies the space coordinate of the previously created avatars and the reconstructed 3D space so that the avatars move within this 3D space to simulate the original event that took place in the input video. This dynamic 3D space can be imported into Unity for the users to explore through other means like AR or VR. We refer the readers to the supplementary video for more details. Thus, IntoTheVideos re-creates the sports event in 3D and users can freely explore the space from various angles beyond the viewpoints in the original footage and even "replay" the event in 3D slow-motion.

\section{DISCUSSION AND FUTURE WORK}

In this work, we presented IntoTheVideo which offers a new way to experience sports events. We reconstruct the 3D space of a sports field/arena from a single video and transform detected players into $3 \mathrm{D}$ avatars to create a more interactive viewing experience. Our system enables a new option to experience sports beyond watching broadcast games or playing video games. Our system has the potential to be more engaging than the former and less demanding than the latter. In the future, we hope to upgrade the texture of the reconstructed 3D space as well as player avatar to match with the video better. Furthermore, the accuracy of the pose estimation is hindered by the size of the bounding box, which leaves room for improvement. Last but not least, we would like to adapt our system on different types of videos beyond sports footage, such 
as game recordings and family gathering recordings, in order to extend this novel immersive recreation to other memorable events.

\section{REFERENCES}

[1] Guilin Liu, Fitsum A Reda, Kevin J Shih, Ting-Chun Wang, Andrew Tao, and Bryan Catanzaro. 2018. Image inpainting for irregular holes using partial convolutions. In Proceedings of the European Conference on Computer Vision (ECCV). 85-100.

[2] Matthew Loper, Naureen Mahmood, Javier Romero, Gerard Pons-Moll, and Michael J. Black. 2015. SMPL: A Skinned Multi-Person Linear Model. ACM Trans. Graphics (Proc. SIGGRAPH Asia) 34, 6 (Oct. 2015), 248:1-248:16.

[3] Celina Ma, Haohong Wang, Hao Sun, Elliot van Huijgevoort, Mea Wang, and Zhihai He. 2021. Powering TV Experiences with Anytime Environmental Exploration In Extended Abstracts of the 2021 CHI Conference on Human Factors in Computing
Systems. 1-6.

[4] NBA. 2021. https://tinyurl.com/3tyverwt.

[5] Dario Pavllo, Christoph Feichtenhofer, David Grangier, and Michael Auli. 2019. 3D human pose estimation in video with temporal convolutions and semi-supervised training. In Conference on Computer Vision and Pattern Recognition (CVPR).

[6] Yuxin Wu, Alexander Kirillov, Francisco Massa, Wan-Yen Lo, and Ross Girshick. 2019. Detectron2. https://github.com/facebookresearch/detectron2.

[7] Wei Yin, Jianming Zhang, Oliver Wang, Simon Niklaus, Long Mai, Simon Chen, and Chunhua Shen. 2021. Learning to Recover 3D Scene Shape from a Single Image. In Proc. IEEE Conf. Comp. Vis. Patt. Recogn. (CVPR).

[8] Yifu Zhang, Chunyu Wang, Xinggang Wang, Wenjun Zeng, and Wenyu Liu. 2020. FairMOT: On the Fairness of Detection and Re-Identification in Multiple Object Tracking. arXiv preprint arXiv:2004.01888 (2020). 